\title{
Androsterone Sulfate
}

National Cancer Institute

\section{Source}

National Cancer Institute. Androsterone Sulfate. NCI Thesaurus. Code C120008.

A sulfated form of androsterone and a steroid metabolite derived from testosterone found in urine, with weak androgenic activity. 